University of Nebraska - Lincoln

DigitalCommons@University of Nebraska - Lincoln

\title{
Persistence of pharmaceutical compounds and other organic wastewater contaminants in a conventional drinking-water- treatment plant
}

\author{
Paul E. Stackelberg \\ U.S. Geological Survey, pestack@usgs.gov \\ Edward T. Furlong \\ U.S. Geological Survey \\ Michael T. Meyer \\ U.S. Geological Survey, mmeyer@usgs.gov \\ Steven D. Zaugg \\ U.S. Geological Survey \\ Alden K. Henderson \\ Centers for Disease Control and Prevention \\ See next page for additional authors \\ Follow this and additional works at: https://digitalcommons.unl.edu/usgsstaffpub \\ Part of the Earth Sciences Commons
}

Stackelberg, Paul E.; Furlong, Edward T.; Meyer, Michael T.; Zaugg, Steven D.; Henderson, Alden K.; and Reissman, Dori B., "Persistence of pharmaceutical compounds and other organic wastewater contaminants in a conventional drinking-water-treatment plant" (2004). USGS Staff -- Published Research. 443.

https://digitalcommons.unl.edu/usgsstaffpub/443

This Article is brought to you for free and open access by the US Geological Survey at DigitalCommons@University of Nebraska - Lincoln. It has been accepted for inclusion in USGS Staff -- Published Research by an authorized administrator of DigitalCommons@University of Nebraska - Lincoln. 


\section{Authors}

Paul E. Stackelberg, Edward T. Furlong, Michael T. Meyer, Steven D. Zaugg, Alden K. Henderson, and Dori B. Reissman 


\title{
Persistence of pharmaceutical compounds and other organic wastewater contaminants in a conventional drinking-water- treatment plant
}

\author{
Paul E. Stackelberga, ${ }^{\mathrm{a}}$, Edward T. Furlong ${ }^{\mathrm{b}}$, Michael T. Meyer ${ }^{\mathrm{c}}$, Steven D. Zaugg ${ }^{\mathrm{b}}$, \\ Alden K. Henderson ${ }^{\mathrm{d}}$, Dori B. Reissman ${ }^{\mathrm{d}}$ \\ ${ }^{a}$ US Geological Survey, 810 Bear Tavern Road, West Trenton, NJ 08628, USA \\ ${ }^{\mathrm{b}}$ US Geological Survey, Box 25046, MS 407, Denver, CO 80225-0046, USA \\ ${ }^{\mathrm{c}}$ US Geological Survey, 4500 SW 40th Avenue, Ocala, FL 34474, USA \\ ${ }^{\mathrm{d}}$ Centers for Disease Control and Prevention, 1600 Clifton Road, MS E23, Atlanta, GA 30333, USA
}

Accepted 18 March 2004

\begin{abstract}
In a study conducted by the US Geological Survey and the Centers for Disease Control and Prevention, 24 water samples were collected at selected locations within a drinking-water-treatment (DWT) facility and from the two streams that serve the facility to evaluate the potential for wastewater-related organic contaminants to survive a conventional treatment process and persist in potable-water supplies. Stream-water samples as well as samples of raw, settled, filtered, and finished water were collected during low-flow conditions, when the discharge of effluent from upstream municipal sewage-treatment plants accounted for $37-67 \%$ of flow in stream 1 and $10-20 \%$ of flow in stream 2. Each sample was analyzed for 106 organic wastewater-related contaminants (OWCs) that represent a diverse group of extensively used chemicals. Forty OWCs were detected in one or more samples of stream water or raw-water supplies in the treatment plant; 34 were detected in more than $10 \%$ of these samples. Several of these compounds also were frequently detected in samples of finished water; these compounds include selected prescription and non-prescription drugs and their metabolites, fragrance compounds, flame retardants and plasticizers, cosmetic compounds, and a solvent. The detection of these compounds suggests that they resist removal through conventional water-treatment processes. Other compounds that also were frequently detected in samples of stream water and rawwater supplies were not detected in samples of finished water; these include selected prescription and non-prescription drugs and their metabolites, disinfectants, detergent metabolites, and plant and animal steroids. The non-detection of these compounds indicates that their concentrations are reduced to levels less than analytical detection limits or that they are transformed to degradates through conventional DWT processes. Concentrations of OWCs detected in finished water generally were low and did not exceed Federal drinking-water standards or lifetime health advisories, although such standards or advisories have not been established for most of these compounds. Also, at least 11 and as many as 17 OWCs were detected in samples of finished water. Drinking-water criteria currently are based on the toxicity of individual compounds and not combinations of compounds. Little is known about potential human-health effects associated with chronic exposure to trace levels of multiple OWCs through routes such as drinking water. The occurrence in drinking-water supplies of many of the OWCs analyzed for during this study is unregulated and most
\end{abstract}

*Corresponding author. Tel.: + 1-609-771-3951; fax: + 1-609-771-3915.

E-mail address: pestack@usgs.gov (P.E. Stackelberg). 
of these compounds have not been routinely monitored for in the Nation's source- or potable-water supplies. This study provides the first documentation that many of these compounds can survive conventional water-treatment processes and occur in potable-water supplies. It thereby provides information that can be used in setting research and regulatory priorities and in designing future monitoring programs. The results of this study also indicate that improvements in water-treatment processes may benefit from consideration of the response of OWCs and other trace organic contaminants to specific physical and chemical treatments.

(c) 2004 Elsevier B.V. All rights reserved.

Keywords: Pharmaceuticals; Wastewater contaminants; Water treatment

\section{Introduction}

During the 1990s, pharmaceutically active compounds such as lipid-regulating drugs, analgesics, antibiotics, antiseptics, hormones, and chemotherapy and beta-blocking heart drugs were detected in wastewaters, streams, and ground-water resources across Europe (Heberer and Stan, 1997; Buser et al., 1998a,b, 1999). Although pharmaceutically active compounds had been detected previously in effluent from landfills (Turner et al., 1993; Holm et al., 1995) and sewage-treatment plants (STPs) (Hignite and Azarnoff, 1977), these more recent investigations indicated that some pharmaceutically active compounds are nearly ubiquitous at low concentrations in water bodies that receive STP effluent. Reviews of the occurrence, fate, and effects of pharmaceutically active compounds in the environment are available (Richardson et al., 1985; Halling-Sorensen et al., 1998; Daughton, 2001; Ternes, 2001).

During 1999-2000, Kolpin et al. (2002) conducted a reconnaissance of pharmaceuticals and other wastewater-related contaminants in susceptible streams across the United States, expanding the scope of previous investigations and providing the first nationwide data set of this type. Results of this reconnaissance survey document that a wide variety of organic compounds are frequently detected in streams that receive agricultural, domestic, and (or) industrial wastewater effluent. These contaminants include antibiotics, other prescription drugs, non-prescription drugs, animal and plant steroids, reproductive hormones, personalcare products, detergent metabolites, flame retardants, products of oil use and combustion, and other extensively used chemicals, collectively referred to as organic wastewater contaminants (OWCs).

Measures to conserve and reuse water commonly are initiated in areas with growing urban populations and constraints on the development of new water sources. These measures include the use of treated municipal wastewater to augment rawwater supplies - a process referred to as indirect potable water reuse (IPWR) (National Research Council, 1998). The presence of OWCs in wastewater effluent is one of the most challenging aspects of IPWR because of the large number of compounds that may be present, the inability to determine all of these compounds, and the lack of toxicity information and drinking-water standards for many of them (National Research Council, 1998). The frequent occurrence of these compounds in streams (Kolpin et al., 2002), some of which are used as sources of drinking water, gives rise to concern over the potential for these compounds to occur in drinking water and, thus, to affect human health through chronic exposure.

To date, few studies have been published concerning the occurrence of OWCs in drinking-water supplies (Kümmerer, 2001). Notable exceptions include the detection of the pharmaceuticals phenazone and propiphenazone and the drug metabolites clofibric acid and 1-acetyl-1-methyl2-dimethyl-oxamoyl-2-phenylhydrazide in samples of potable water collected in the vicinity of Berlin, Germany (Heberer and Stan, 1997; Reddersen et al., 2002), and the detection of three widely used non-prescription drugs-caffeine, cotinine, and acetaminophen-in samples of potable water collected near Atlanta, Georgia (Frick et al., 2001).

The objective of this study, conducted by the US Geological Survey (USGS) and the Centers for Disease Control and Prevention, was to build 
on the above-mentioned investigations by examining a wider variety of OWCs in order to further assess the potential for these compounds to survive conventional water treatment and occur in finished-water supplies. The occurrence of many of these compounds in drinking water is unregulated and most of them have not been routinely monitored for in the Nation's source- and potable-water supplies. By documenting the occurrence of a broad suite of OWCs in source- and potable-water supplies, results of this study can be used in setting monitoring, research, and regulatory priorities, and in designing appropriate chemical and toxicological studies and risk assessments to address more fully the potential health effects associated with IPWR.

This paper documents the occurrence of select OWCs in 24 samples of stream, raw, settled, filtered, and finished water associated with a drinking-water-treatment (DWT) facility. Although these OWCs represent a diverse group of chemicals with a wide variety of uses, they account for only a fraction of the thousands of organic compounds that currently are manufactured and used for therapeutic purposes or that occur in various consumer and household, industrial and commercial, or agricultural products. This study, therefore, does not provide information on all of the OWCs that may occur in source- or potable-water supplies; rather, it documents the occurrence of selected organic contaminants representing a wide variety of uses and origins in environmental waters and in potable-water supplies.

\section{Description of site and sampling methods}

The DWT facility from which samples were collected during this study is in a heavily populated, highly urbanized drainage basin. More than 50 STPs discharge effluent to the two streams from which the DWT facility withdraws its raw-water supplies or to tributaries of these streams. Samples of stream water were collected from the streams that provide raw water to the DWT facility (Fig. 1a). USGS personnel collected all stream samples using standard depth- and width-integrating tech- niques (Shelton, 1994) and water-quality sampling field protocols (US Geological Survey, 1998). At each site, a composite sample of unfiltered water was collected from approximately 10 vertical profiles and then split into baked, 1-liter (1) amber glass bottles that were immediately chilled and shipped to participating laboratories. The sampling site on stream 2 was located approximately 30 meters $(\mathrm{m})$ upstream from a source-water intake (Fig. 1a). Stream 2 flows into stream 1 approximately $6175 \mathrm{~m}$ upstream from a source-water intake on stream 1. In order to distinguish the chemical signature of each stream, the sampling site for stream 1 was located just upstream from their confluence (Fig. 1a). This design allows for the identification of differences in chemical occurrence associated with each stream but does not account for sources that may be present between their confluence and the source-water intake on stream 1. A STP located along this reach of stream 1 discharges an average of 7 million gallons per day (Mgal/d) (Fig. 1a).

The DWT facility treats an average of $62 \mathrm{Mgal} /$ $\mathrm{d}$, providing potable water to an estimated 850000 people. The facility utilizes a conventional treatment process that consists of the following sequence of physical and chemical treatments: (1) raw-water screening - the movement of raw water past a stationary bar rack and two traveling screens to remove coarse debris; (2) the addition of powdered activated carbon to remove taste- and odorcausing compounds as well as organic chemicals; (3) the addition of sulfuric acid or caustic for $\mathrm{pH}$ control; (4) coagulation - the addition of coagulant salts and polymers to destabilize colloidal particles and facilitate their flocculation with other suspended particles; (5) primary disinfection - the addition of Na hypochlorite to inactivate pathogenic microorganisms; (6) flocculation - the agitation of coagulated water to promote the aggregation of suspended materials; (7) sedimentation - the stilling of flocculated water to promote the settling of suspended solids and floccules; (8) filtration - the movement of water through tanks that contain sand and either bituminous granular activated carbon (GAC), lignite GAC, or anthracite to retain remaining fine solids and bacteria; (9) secondary disinfection - the addition of $\mathrm{Na}$ hypochlorite to 


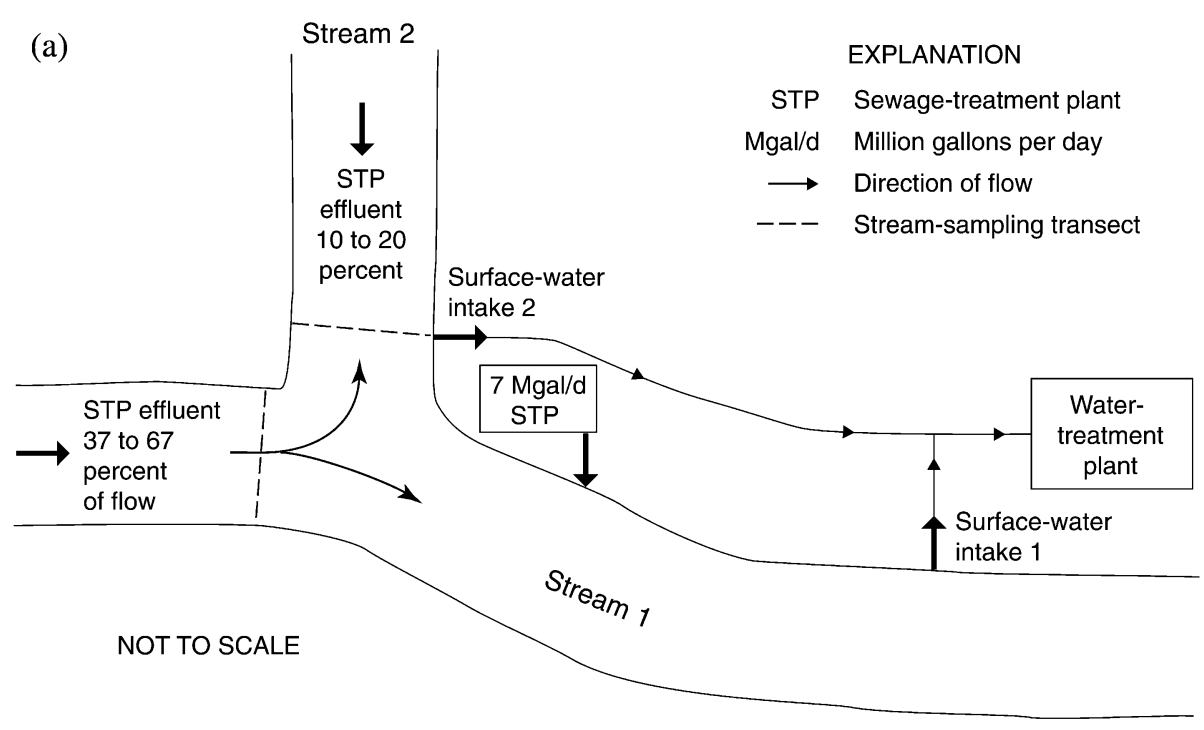

(b)

EXPLANATION

- Water-treatment plant sampling location

PAC Powdered activated carbon

$\longrightarrow$ Direction of flow

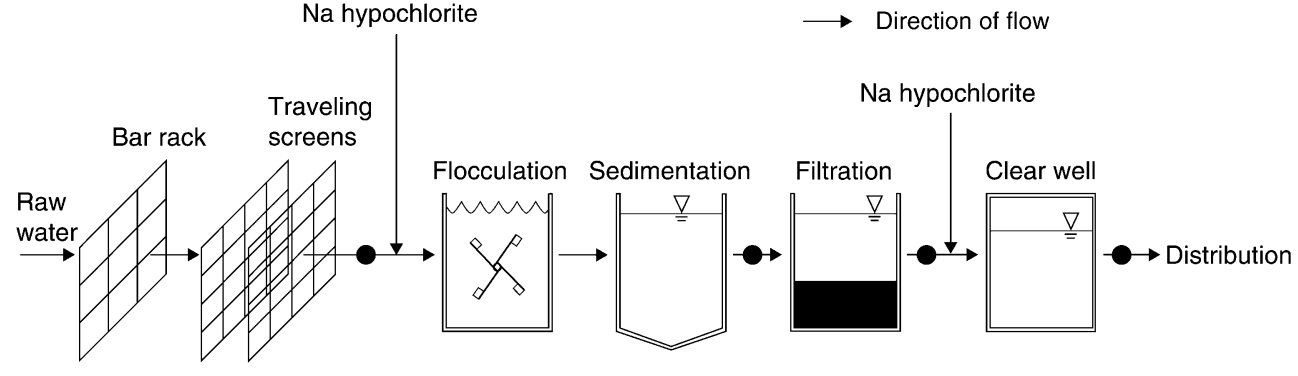

NOT TO SCALE

Fig. 1. Schematic diagram showing (a) location of stream sampling sites, surface-water intakes, and drinking-water-treatment plant, and (b) physical and chemical processes used in drinking-water-treatment plant.

maintain a chlorine residual in the distribution system; and (10) the addition of caustic soda to maintain a $\mathrm{pH}$ of 7.8 to 8.2 for corrosion control (Fig. 1b).

Samples of unfiltered water were collected at selected locations in the DWT facility; raw water, settled water, filtered water, and finished water were collected (Fig. 1b). Plant personnel collected the DWT-facility samples by filling baked, 1-1 amber glass bottles from spigots. These samples were chilled immediately and sent to participating laboratories.
All samples were collected over 4 consecutive weeks during November and December 2001. Stream samples were collected later than samples of raw water in the DWT facility and the collection of samples in the DWT facility did not completely account for expected retention times associated with each treatment process. To the extent that the occurrence of some OWCs in raw-water supplies may be transient in nature, the timing of sample collection may introduce some degree of uncertainty into the results of this study. Nonetheless, the design of this study allows for an initial 
assessment of the occurrence and concentration of OWCs in water at various points before, during, and after treatment.

\section{Analytical methods and quality assurance}

One hundred six compounds were analyzed for in each sample using three analytical methods recently developed by the USGS (Table 1). Four compounds (sulfamethoxazole, trimethoprim, caffeine, and cotinine) were measured by more than one method. Descriptions of the analytical methods and method performance characteristics are provided elsewhere (Barber et al., 2000; Kolpin et al., 2002; Cahill et al., 2004). The list of analytes and their reporting levels reported herein differ slightly from those in Kolpin et al. (2002) as a result of additions to the list of analytes and increases in method reporting levels for the wastewater-related compounds by continuous liquid-liquid extraction and gas chromatography/mass spectrometry (CLLE GC/MS). Twenty-five antibiotic compounds were extracted and analyzed by tandem solid-phase extraction (SPE) and single quadrapole, liquid chromatography/mass spectrometry with electro-spray ionization set in positive mode and selected-ion monitoring (SIM). Reporting levels for the antibiotic method range from 0.02 to $2.0 \mu \mathrm{g} / 1$ (Table 1). Twenty-two human prescription and non-prescription drugs and selected metabolites were extracted by SPE and measured by high performance liquid chromatography/electrospray-ionization mass spectrometry (HPLC/ ESI-MS) using a reverse-phase octylsilane (C8) HPLC column (Cahill et al., 2004). Reporting levels for the prescription and non-prescription drugs method range from 0.001 to $0.24 \mu \mathrm{g} / 1$ (Table 1). Sixty-three other OWCs were extracted from whole-water using CLLE GC/MS (Barber et al., 2000). Reporting levels for the wastewaterrelated compounds method range from 0.5 to 5.0 $\mu \mathrm{g} / 1$ (Table 1). For each method, concentrations of constituents with confirmed detections below the reporting level are provided. The uncertainty associated with concentrations reported below the reporting limit is greater than concentrations reported above the reporting limit; therefore, concentrations below the reporting limit are reported as estimates. Method recovery and precision data are reported in Barber et al. (2000) and Cahill et al. (2004).

One field and 27 laboratory blanks were analyzed for target compounds during the course of this study. Blank samples were derived from laboratory-grade organic-free water and were used to determine whether sampling procedures, sampling equipment, field conditions, or sample shipment procedures introduced the target analytes to environmental samples (field blank) or to assess the potential for sample contamination in the laboratory (laboratory blanks). Carbamazepine, sulfamethoxazole, and trimethoprim were each detected in the field blank at $0.0001 \mu \mathrm{g} / 1$ for trimethoprim, $0.0003 \mu \mathrm{g} / 1$ for carbamazepine, and $0.0009 \mu \mathrm{g} / 1$ for sulfamethoxazole. These concentrations are well below the reporting levels for these compounds (Table 1) and reflect (1) the sensitivity for detecting these compounds by HPLC/ESI-MS; and (2) possible low-level carryover between instrumental analysis. Carbamazepine was detected in the associated environmental sample, but at a concentration more than 100 times that measured in the field blank. Sulfamethoxazole and trimethoprim were not detected in the associated environmental sample. Moreover, sulfamethoxazole was detected in only one of the 24 samples collected. Although carbamazepine and trimethoprim were detected more frequently, concentrations of these compounds in stream samples and samples from the DWT facility were at least 20 times greater than concentrations observed in the field blank.

Indole and diphenhydramine were each detected in one laboratory blank. Indole was not detected in any of the associated environmental samples, whereas diphenhydramine was detected in one associated environmental sample, but at a concentration 10 times that measured in the laboratory blank. Erythromycin- $\mathrm{H}_{2} \mathrm{O}$ was detected at concentrations less than $0.01 \mu \mathrm{g} / 1$ in laboratory blanks. Erythromycin is a low-level contaminant in the ${ }^{13} \mathrm{C}$-erythromycin surrogate standard and is converted to erythromycin- $\mathrm{H}_{2} \mathrm{O}$ when the sample is acidified. Environmental concentrations of erythromycin- $\mathrm{H}_{2} \mathrm{O}$ reported herein are more than three times the observed background concentration in laboratory blanks. The remaining target analytes 
Table 1

Wastewater-related compounds analyzed for in all samples and highest concentration detected in samples of finished water

\begin{tabular}{|c|c|c|c|c|c|c|c|}
\hline Chemical & $\begin{array}{l}\text { CAS } \\
\text { number }\end{array}$ & General use & $\begin{array}{l}\text { Reporting } \\
\text { level } \\
(\mu \mathrm{g} / 1)\end{array}$ & $\begin{array}{l}\text { Detection } \\
\text { frequency } \\
\text { in stream } \\
\text { and raw- } \\
\text { water samples } \\
(n=12)\end{array}$ & $\begin{array}{l}\text { Drinking } \\
\text { water } \\
\text { standards } \\
\text { and health } \\
\text { advisories } \\
(\mu \mathrm{g} / 1)\end{array}$ & $\begin{array}{l}\text { Cancer } \\
\text { group }\end{array}$ & $\begin{array}{l}\text { Highest } \\
\text { concentration } \\
\text { in sample of } \\
\text { finished } \\
\text { water } \\
(\mu \mathrm{g} / 1)\end{array}$ \\
\hline \multicolumn{8}{|l|}{ Antibiotics by LCMS } \\
\hline Carbadox & $6804-07-5$ & Antibiotic & 0.10 & 0 & - & - & ND \\
\hline Chlortetracycline & $57-62-5$ & Antibiotic & 0.05 & 0 & - & - & ND \\
\hline Ciprofloxacin & $85721-33-1$ & Antibiotic & 0.02 & 0 & - & - & ND \\
\hline Demeclocycline & $127-33-3$ & Antibiotic & 0.05 & 0 & - & - & $\mathrm{ND}$ \\
\hline Doxycycline & $564-25-0$ & Antibiotic & 0.1 & 0 & - & - & ND \\
\hline Enrofloxacin & $93106-60-6$ & Antibiotic & 0.02 & 0 & - & - & ND \\
\hline Erythromycin $-\mathrm{H}_{2} \mathrm{O}$ & - & $\begin{array}{l}\text { Erythromycin } \\
\text { metabolite }\end{array}$ & 0.05 & 67 & - & - & ND \\
\hline Lincomycin & $154-21-2$ & Antibiotic & 0.05 & 0 & - & - & $\mathrm{ND}$ \\
\hline Methotrexate & $59-05-2$ & Antibiotic & 0.05 & 0 & - & - & ND \\
\hline Minocycline & $10118-90-8$ & Antibiotic & 0.05 & 0 & - & - & $\mathrm{ND}$ \\
\hline Norfloxacin & $70458-96-7$ & Antibiotic & 0.02 & 0 & - & - & ND \\
\hline Oxytetracycline & $79-57-2$ & Antibiotic & 0.1 & 0 & - & - & ND \\
\hline Roxarsone & $121-19-7$ & Antibiotic & 2.0 & 0 & - & - & $\mathrm{ND}$ \\
\hline Roxithromycin & $80214-83-1$ & Antibiotic & 0.03 & 0 & - & - & ND \\
\hline Sarafloxacin & $98105-99-8$ & Antibiotic & 0.02 & 0 & - & - & ND \\
\hline Sulfadimethoxine & $122-11-2$ & Antibiotic & 0.05 & 0 & - & - & ND \\
\hline Sulfamerazine & $127-79-7$ & Antibiotic & 0.05 & 0 & - & - & ND \\
\hline Sulfamethazine & $57-68-1$ & Antibiotic & 0.05 & 0 & - & - & ND \\
\hline Sulfamethizole & $144-82-1$ & Antibiotic & 0.05 & 0 & - & - & $\mathrm{ND}$ \\
\hline Sulfamethoxazole ${ }^{1}$ & $723-46-6$ & Antibiotic & 0.05 & 8 & - & - & ND \\
\hline Sulfathiazole & $72-14-0$ & Antibiotic & 0.10 & 0 & - & - & $\mathrm{ND}$ \\
\hline Tetracycline & $60-54-8$ & Antibiotic & 0.05 & 0 & - & - & ND \\
\hline Trimethoprim ${ }^{1}$ & $738-70-5$ & Antibiotic & 0.03 & 0 & - & - & $\mathrm{ND}$ \\
\hline Tylosin & $1401-69-0$ & Antibiotic & 0.05 & 0 & - & - & $\mathrm{ND}$ \\
\hline Virginiamycin & 21411-53-0 & Antibiotic & 0.10 & 0 & - & - & $\mathrm{ND}$ \\
\hline \multicolumn{8}{|c|}{$\begin{array}{l}\text { Prescription and non-prescription drugs by } \\
\quad \text { HPLC/ESI-MS }\end{array}$} \\
\hline 1,7-dimethylxanthine & $611-59-6$ & Caffeine metabolite & 0.018 & 75 & - & - & ND \\
\hline Acetaminophen & $103-90-2$ & Antipyretic & 0.009 & 50 & - & - & ND \\
\hline Albuterol & $18559-94-9$ & Antiasthmatic & 0.029 & 8 & - & - & $\mathrm{ND}$ \\
\hline Caffeine $^{2}$ & $58-08-2$ & Stimulant & 0.014 & 100 & - & - & 0.119 \\
\hline Carbamazepine & $298-46-4$ & Anticonvulsant & 0.011 & 100 & - & - & 0.258 \\
\hline Cimetidine & $51481-61-9$ & Antacid & 0.007 & 0 & - & - & ND \\
\hline
\end{tabular}




\begin{tabular}{|c|c|c|c|c|c|c|c|}
\hline Chemical & $\begin{array}{l}\text { CAS } \\
\text { number }\end{array}$ & General use & $\begin{array}{l}\text { Reporting } \\
\text { level } \\
(\mu \mathrm{g} / 1)\end{array}$ & $\begin{array}{l}\text { Detection } \\
\text { frequency } \\
\text { in stream } \\
\text { and raw- } \\
\text { water samples } \\
(n=12)\end{array}$ & $\begin{array}{l}\text { Drinking } \\
\text { water } \\
\text { standards } \\
\text { and health } \\
\text { advisories } \\
(\mu \mathrm{g} / 1)\end{array}$ & $\begin{array}{l}\text { Cancer } \\
\text { group }\end{array}$ & $\begin{array}{l}\text { Highest } \\
\text { concentration } \\
\text { in sample of } \\
\text { finished } \\
\text { water } \\
(\mu \mathrm{g} / 1)\end{array}$ \\
\hline Codeine & $76-57-3$ & Analgesic & 0.24 & 25 & - & - & ND \\
\hline Cotinine $^{2}$ & $486-56-6$ & Nicotine metabolite & 0.023 & 100 & - & - & 0.025 \\
\hline Dehydronifedipine & 67035-22-7 & Nifedipine metabolite & 0.01 & 50 & - & - & 0.004 \\
\hline Digoxigenin & $1672-46-4$ & Digoxin metabolite & 0.008 & 0 & - & - & ND \\
\hline Diltiazem & $42399-41-7$ & Antihypertensive & 0.012 & 0 & - & - & ND \\
\hline Diphenhydramine & $58-73-1$ & Antihistamine & 0.0148 & 25 & - & - & ND \\
\hline Fluoxetine & $54910-89-3$ & Antidepressant & 0.018 & 0 & - & - & ND \\
\hline Furosemide & $54-31-9$ & Diuretic & 0.0386 & 0 & - & - & ND \\
\hline Gemfibrozil & $25812-30-0$ & Antihyperlipidemic & 0.015 & 0 & - & - & ND \\
\hline Ibuprofen & $15687-27-1$ & Antiinflammatory & 0.018 & 0 & - & - & ND \\
\hline Miconazole & $22916-47-8$ & Antifungal & 0.0175 & 0 & - & - & ND \\
\hline Ranitidine & $66357-35-5$ & Antacid & 0.01 & 0 & - & - & ND \\
\hline Sulfamethoxazole $^{1}$ & $723-46-6$ & Antibiotic & 0.023 & 8 & - & - & ND \\
\hline Thiabendazole & $148-79-8$ & Fungicide & 0.0108 & 8 & - & - & ND \\
\hline Trimethoprim $^{1}$ & $738-70-5$ & Antibiotic & 0.014 & 83 & - & - & ND \\
\hline Warfarin & $81-81-2$ & Anticoagulant & 0.001 & 0 & - & - & ND \\
\hline \multicolumn{8}{|l|}{$\begin{array}{l}\text { Other wastewater-related compounds by } \\
\qquad L L E G C / M S\end{array}$} \\
\hline 1,4-dichlorobenzene & $106-46-7$ & Deodorizer & 0.5 & 0 & $75^{3} ; 75^{4} ; 0.1^{5} ; 4000^{6}$ & $\mathrm{C}$ & ND \\
\hline 1-methylnapthalene & $90-12-0$ & Fuels & 0.5 & 0 & - & - & ND \\
\hline 2,6-dimethylnapthalene & $581-42-0$ & Fuels & 0.5 & 0 & - & - & ND \\
\hline 2-methylnapthalene & $91-57-6$ & Fuels & 0.5 & 0 & - & - & ND \\
\hline 3- $\beta$-coprostanol & $360-68-9$ & Fecal steroid & 2 & 33 & - & - & ND \\
\hline 3-methyl-1H-indole (skatol) & $83-34-1$ & Fragrance & 1 & 0 & - & - & ND \\
\hline 3-tert-butyl-4-hydroxyanisole (BHA) & $25013-16-5$ & Antioxidant & 5 & 0 & - & - & ND \\
\hline 4-cumylphenol & $599-64-4$ & Detergent metabolite & 1 & 0 & - & - & ND \\
\hline 4-n-octylphenol & $1806-26-4$ & Detergent metabolite & 1 & 0 & - & - & ND \\
\hline 4-nonylphenol diethoxylate (NPEO2-total) & $26027-38-3$ & Detergent metabolite & 5 & 58 & - & - & ND \\
\hline 4-octylphenol diethoxylate (OPEO2) & $26636-32-8$ & Detergent metabolite & 1 & 0 & - & - & ND \\
\hline 4-octylphenol monoethoxylate (OPEO1) & $26636-32-8$ & Detergent metabolite & 1 & 0 & - & - & ND \\
\hline 4-tert-octylphenol & $140-66-9$ & Detergent metabolite & 1 & 8 & - & - & ND \\
\hline 5-methyl-1H-benzotriazole & $136-85-6$ & Anticorrosive & 2 & 0 & - & - & ND \\
\hline Acetophenone & $98-86-2$ & fragrance & 0.5 & 0 & - & - & ND \\
\hline $\begin{array}{l}\text { 7-acetyl-1,1,3,4,4,6-hexamethyl tetrahydronaphthalene } \\
\text { (AHTN) }\end{array}$ & $21145-77-7$ & Fragrance & 0.5 & 100 & - & - & 0.49 \\
\hline Anthracene & $120-12-7$ & PAH & 0.5 & 0 & $0.3^{5} ; 10000^{6}$ & $\mathrm{D}$ & ND \\
\hline Anthraquinone & $84-65-1$ & Manufacturing & 0.5 & 42 & - & - & 0.072 \\
\hline
\end{tabular}




\begin{tabular}{|c|c|c|c|c|c|c|c|}
\hline Chemical & $\begin{array}{l}\text { CAS } \\
\text { number }\end{array}$ & General use & $\begin{array}{l}\text { Reporting } \\
\text { level } \\
(\mu \mathrm{g} / 1)\end{array}$ & $\begin{array}{l}\text { Detection } \\
\text { frequency } \\
\text { in stream } \\
\text { and raw- } \\
\text { water samples } \\
(n=12)\end{array}$ & $\begin{array}{l}\text { Drinking } \\
\text { water } \\
\text { standards } \\
\text { and health } \\
\text { advisories } \\
(\mu \mathrm{g} / 1)\end{array}$ & $\begin{array}{l}\text { Cancer } \\
\text { group }\end{array}$ & $\begin{array}{l}\text { Highest } \\
\text { concentration } \\
\text { in sample of } \\
\text { finished } \\
\text { water } \\
(\mu \mathrm{g} / 1)\end{array}$ \\
\hline Benzo $[a]$ pyrene & $50-32-8$ & PAH & 0.5 & 42 & $0.2^{3}$ & B2 & ND \\
\hline Benzophenone & $119-61-9$ & Fixative & 0.5 & 58 & - & - & 0.13 \\
\hline$\beta$-sitosterol & $83-46-5$ & Plant steroid & 2 & 83 & - & - & ND \\
\hline$\beta$-stigmastanol & $19466-47-8$ & Plant steroid & 2 & 8 & - & - & ND \\
\hline Bisphenol A & $80-05-7$ & Plasticizer & 1 & 100 & - & - & 0.42 \\
\hline Bromacil & $314-40-9$ & Herbicide & 0.5 & 0 & $90^{4} ; 0.1^{5} ; 5000^{6}$ & $\mathrm{C}$ & ND \\
\hline Bromoform $^{7}$ & $75-25-2$ & Trihalomethane & 0.5 & 50 & $80^{3} ; 0.02^{5} ; 700^{6}$ & B2 & 21 \\
\hline Caffeine $^{2}$ & $58-08-2$ & Stimulant & 0.5 & 100 & - & - & ND \\
\hline Camphor & $76-22-2$ & Flavorant & 0.5 & 0 & - & - & ND \\
\hline Carbaryl & $63-25-2$ & Insecticide & 1 & 0 & $700^{4} ; 0.1^{5} ; 4000^{6}$ & $\mathrm{D}$ & ND \\
\hline Carbazole & $86-74-8$ & Insecticide & 0.5 & 17 & - & - & ND \\
\hline Chlorpyrifos & $2921-88-2$ & Insecticide & 0.5 & 0 & $20^{4} ; 0.003^{5} ; 100^{6}$ & $\mathrm{D}$ & ND \\
\hline Cholesterol & $57-88-5$ & Plant/animal steroid & 2 & 83 & - & - & ND \\
\hline Cotinine $^{2}$ & $486-56-6$ & Nicotine metabolite & 1 & 0 & - & - & ND \\
\hline Diazinon & $333-41-5$ & Insecticide & 0.5 & 0 & - & - & ND \\
\hline$d$-dichlorvos & $62-73-7$ & Insecticide & 1 & 0 & - & - & ND \\
\hline$d$-limonene & $5989-27-5$ & Fungicide & 0.5 & 0 & - & - & ND \\
\hline Fluoranthene & $206-44-0$ & $\mathrm{PAH}$ & 0.5 & 8 & - & - & ND \\
\hline 1,3,4,6,7,8-hexahydro-4,6,6,7,8,8-hexamethyl & $1222-05-5$ & Fragrance & 0.5 & 92 & - & - & 0.082 \\
\hline \multicolumn{8}{|l|}{ Cyclopenta- $\gamma$-2-benzopyran (HHCB) } \\
\hline Indole & $120-72-9$ & Pesticide inert & 0.5 & 0 & - & - & ND \\
\hline Isoborneol & $124-76-5$ & Fragrance & 0.5 & 0 & - & - & ND \\
\hline Isophorone & $78-59-1$ & Solvent & 0.5 & 0 & $100^{4} ; 0.2^{5} ; 7000^{6}$ & $\mathrm{C}$ & ND \\
\hline Isopropylbenzene (cumene) & $98-82-8$ & Fuels & 0.5 & 0 & $0.1^{5} ; 4000^{6}$ & $\mathrm{D}$ & ND \\
\hline Isoquinoline & $119-65-3$ & Flavorant & 0.5 & 0 & - & - & ND \\
\hline Menthol & $89-78-1$ & Fragrance & 0.5 & 0 & - & - & ND \\
\hline Metalaxyl & $57837-19-1$ & Fungicide & 0.5 & 0 & - & - & ND \\
\hline Methyl salicylate & $119-36-8$ & Liniment & 0.5 & 0 & - & - & ND \\
\hline Metolachlor & $51218-45-2$ & Herbicide & 0.5 & 0 & $100^{4} ; 0.15^{5} ; 500^{6}$ & $\mathrm{C}$ & ND \\
\hline$N, N$-diethyl-meta-toluamide (DEET) & $134-62-3$ & Insecticide & 0.5 & 25 & - & - & 0.066 \\
\hline Naphthalene & $91-20-3$ & $\mathrm{PAH}$ & 0.5 & 0 & $100^{4} ; 0.02^{5} ; 700^{6}$ & $\mathrm{C}$ & ND \\
\hline Para-cresol & $106-44-5$ & Wood preservative & 1 & 0 & - & - & ND \\
\hline Para-nonylphenol (total, NP) & $84852-15-3$ & Detergent metabolite & 5 & 0 & - & - & ND \\
\hline Pentachlorophenol & $87-86-5$ & Wood preservative & 2 & 33 & $1^{3} ; 0.03^{5} ; 1000^{6}$ & B2 & ND \\
\hline Phenanthrene & $85-01-8$ & PAH & 0.5 & 0 & & $\mathrm{D}$ & ND \\
\hline Phenol & $108-95-2$ & Disinfectant & 0.5 & 67 & $4000^{4} ; 0.6^{5} ; 20000^{6}$ & $\mathrm{D}$ & ND \\
\hline Prometon & $1610-18-0$ & Herbicide & 0.5 & 25 & $100^{4} ; 0.015^{5} ; 500^{6}$ & $\mathrm{D}$ & 0.096 \\
\hline
\end{tabular}




\begin{tabular}{|c|c|c|c|c|c|c|c|}
\hline Chemical & $\begin{array}{l}\text { CAS } \\
\text { number }\end{array}$ & General use & $\begin{array}{l}\text { Reporting } \\
\text { level } \\
(\mu \mathrm{g} / 1)\end{array}$ & $\begin{array}{l}\text { Detection } \\
\text { frequency } \\
\text { in stream } \\
\text { and raw- } \\
\text { water samples } \\
(n=12)\end{array}$ & $\begin{array}{l}\text { Drinking } \\
\text { water } \\
\text { standards } \\
\text { and health } \\
\text { advisories } \\
(\mu \mathrm{g} / 1)\end{array}$ & $\begin{array}{l}\text { Cancer } \\
\text { group }\end{array}$ & $\begin{array}{l}\text { Highest } \\
\text { concentration } \\
\text { in sample of } \\
\text { finished } \\
\text { water } \\
(\mu \mathrm{g} / 1)\end{array}$ \\
\hline Pyrene & $129-00-0$ & PAH & 0.5 & 8 & $0.03^{5}$ & $\mathrm{D}$ & ND \\
\hline Tetrachloroethylene & $127-18-4$ & Solvent & 0.5 & 58 & $5^{3} ; 10^{4} ; 0.01^{5} ; 500^{6}$ & - & 0.1 \\
\hline Tri(2-butoxyethyl) phosphate & $78-51-3$ & Plasticizer & 0.5 & 83 & - & - & 0.35 \\
\hline Tri(2-chloroethyl) phosphate & $115-96-8$ & Flame retardant & 0.5 & 100 & - & - & 0.099 \\
\hline Tri(dichlorisopropyl) phosphate & $13674-87-8$ & Flame retardant & 0.5 & 100 & - & - & 0.25 \\
\hline Tributyl phosphate & $126-73-8$ & Flame retardant & 0.5 & 83 & - & - & 0.1 \\
\hline Triclosan & $3380-34-5$ & Antimicrobial disinfectant & 1 & 67 & - & - & ND \\
\hline Triethyl citrate (ethyl citrate) & $77-93-0$ & Cosmetics & 0.5 & 50 & - & - & 0.062 \\
\hline Triphenyl phosphate & $115-86-6$ & Plasticizer & 0.5 & 0 & - & - & ND \\
\hline
\end{tabular}

CAS number: chemical abstract service number; B2: probable human carcinogen (US Environmental Protection Agency, 2002); C: possible human carcinogen (US Environmental Protection Agency, 2002); D: not classifiable as to human carcinogenicity (US Environmental Protection Agency, 2002); LCMS: liquid chromatography/ electrospray ionization mass spectrometry; HPLC/ESI-MS: high performance liquid chromatography/mass spectrometry; CLLE GC/MS: continuous liquid - liquid extraction with gas chromatography/mass spectrometry; PAH; polycyclic aromatic hydrocarbon; ND: not detected; -: no data; compounds suspected of being hormonally active are in bold.

${ }^{1}$ Compound analyzed by LCMS and HPLC/ESI-MS.

${ }^{2}$ Compound analyzed by HPLC/ESI-MS and CLLE GC/MS.

${ }^{3}$ US Environmental Protection Agency maximum contaminant level ( $\left.\mu \mathrm{g} / 1\right)$ (US Environmental Protection Agency, 2002).

${ }^{4}$ US Environmental Protection Agency lifetime health advisory ( $\left.\mu \mathrm{g} / \mathrm{l}\right)$ (US Environmental Protection Agency, 2002).

${ }^{5}$ US Environmental Protection Agency reference dose (mg/kg/day) (US Environmental Protection Agency, 2002).

${ }^{6}$ US Environmental Protection Agency drinking water equivalent level ( $\left.\mu \mathrm{g} / \mathrm{l}\right)$ (US Environmental Protection Agency, 2002).

${ }^{7} 1998$ final rule for disinfection by-products: the total for trihalomethanes is $80 \mu \mathrm{g} / \mathrm{l}$ (US Environmental Protection Agency, 2002). 
were not detected in any of the laboratory blanks. These results indicate that sample-collection procedures, sampling equipment, field conditions, and laboratory procedures did not systematically introduce any of the target compounds into field samples at concentrations relevant to observed environmental concentrations.

\section{Results}

Stream samples were collected during low-flow conditions. Long-term mean daily flows at three nearby gaging stations on the two source streams were compared to flow rates at the gaging stations at the time of stream-sample collection. On the basis of 22-115 years of record, flow rates at the gaging stations ranged from as little as approximately $3 \%$ to no more than approximately $30 \%$ of long-term mean daily values. On the basis of average seasonal volumes of STP effluent in each stream and flow rates at the time of sample collection, 37-67\% of the flow in stream 1 and $10-20 \%$ of the flow in stream 2 consisted of STP effluent (Fig. 1a). Furthermore, during such lowflow conditions, some of the flow in stream 1 is diverted into stream 2 to accommodate the volume of water withdrawn at surface-water intake 2 (Fig. 1a). Results reported here pertain only to these conditions and should not be assumed to be representative of water-quality conditions during higher flow conditions.

Forty compounds were detected in samples of stream water or raw-water supplies in the DWT plant; 34 were detected in more than $10 \%$ of these samples (Fig. 2). Seven compounds were detected in each sample of stream water and raw-water supplies: 7-acetyl-1,1,3,4,4,6-hexamethyl tetrahydronaphthalene (AHTN) (a fragrance compound), bisphenol A (a compound used as a manufacturing intermediate, as a component in flame retardants and rubber chemicals, and as a fungicide), caffeine (a stimulant found in coffee, tea, and other beverages), carbamazepine (an anticonvulsant and specific analgesic for trigeminal neuralgia), cotinine (a metabolite of nicotine), tri(2-chloroethyl) phosphate (a flame retardant and plasticizer), and tri(dichloroisopropyl) phosphate (a flame retardant) (Fig. 2). Compounds detected in $75 \%$ or more of these samples are 1,3,4,6,7,8-hexahydro$4,6,6,7,8,8$-hexamethyl cyclopenta- $\gamma$-2-benzopyran (HHCB) (a fragrance compound), $\beta$-sitosterol and cholesterol (plant and animal steroids), tri(2butoxyethyl) phosphate (a plasticizer and flame retardant), tributyl phosphate (a plasticizer), trimethoprim (an antibiotic), and 1,7-dimethylxanthine (a metabolite of caffeine) (Fig. 2). Many of these compounds likely are derived from domestic and (or) industrial wastewaters that are processed through municipal STPs. These facilities, however, generally are designed to remove suspended solids and oxygen-demanding substances and, for advanced sewage treatment, to remove dissolved inorganic constituents such as phosphate. STPs are not specifically designed to remove organic contaminants that are likely to be present in domestic and industrial wastewaters at trace levels. Low concentrations of these OWCs, therefore, are likely to be present in effluent from municipal STPs; the incomplete removal of pharmaceuticals from wastewater has previously been documented (Stumpf et al., 1999; Heberer, 2002). Although most of the more frequently detected organic compounds likely are derived from domestic and (or) industrial wastewaters, some compounds could be derived from non-point sources or sources unrelated to human activities.

Several compounds that were frequently detected in samples of stream water and raw-water supplies also were frequently detected in samples collected throughout the DWT plant, indicating that these compounds resist removal through conventional water-treatment processes (Fig. 3). The concentrations of several compounds (e.g. HHCB, tetrachloroethylene, tri(2-chloroethyl) phosphate, and tributyl phosphate) are consistent across sampling events and throughout the treatment process, suggesting temporally constant concentrations in the source streams and little or no removal through conventional water treatment (Fig. 3). The consistent concentrations of these compounds across sampling events corroborate analytical method performance as described elsewhere (Barber et al., 2000; Kolpin et al., 2002; Cahill et al., 2004). The concentrations of other compounds (e.g. AHTN, bisphenol A, caffeine, and carbamazepine) are more variable across sampling events and through- 


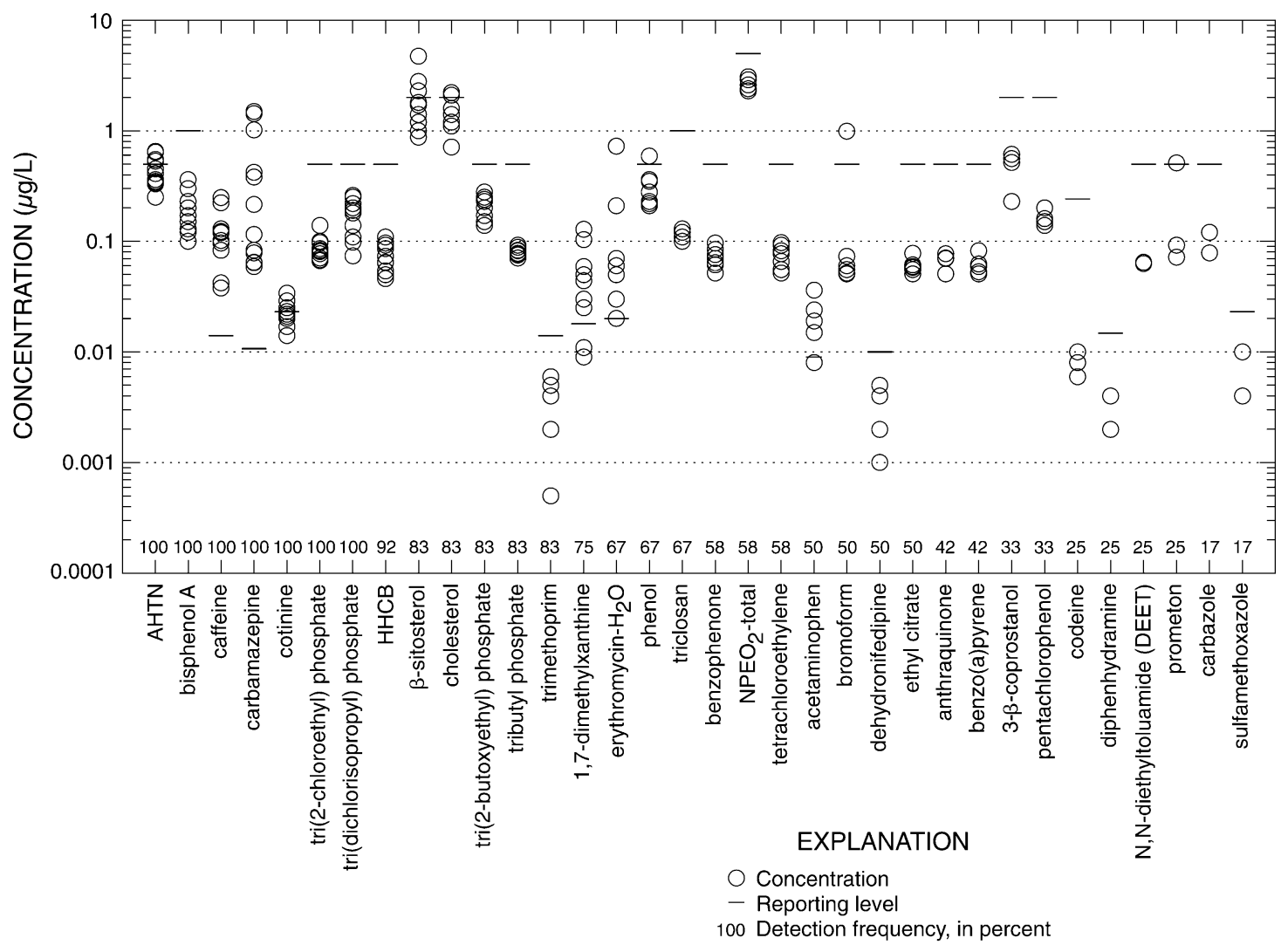

Fig. 2. Concentrations and frequency of detection of compounds detected in more than $10 \%$ of samples of stream water and rawwater supplies in the drinking-water-treatment plant.

out the treatment process, indicating that the concentrations of these compounds in the source streams are temporally more variable and, possibly, that the concentrations of these compounds may be reduced through the DWT process (Fig. 3). The concentrations of these compounds in finished water (Fig. 4) generally were similar to or less than concentrations in the associated raw-water sample, indicating that the variability in concentrations of compounds such as AHTN, bisphenol A, caffeine, and carbamazepine throughout the treatment process shown in Fig. 3 is at least partly due to a reduction in concentration along the treatment process.

Other compounds frequently detected in samples of stream water and raw-water supplies (e.g. cholesterol, $\beta$-sitosterol, trimethoprim, 1,7-dimethy- lxanthine, erythromycin $-\mathrm{H}_{2} \mathrm{O}$, triclosan, phenol, 4-nonylphenol diethoxylate, and acetaminophen) were not detected in samples of finished water, indicating that concentrations of these compounds are effectively reduced to levels less than analytical detection limits or that the compounds are transformed through conventional water-treatment processes to degradates not determined by the methods used in this study.

Previous investigators have reported that filtration with GAC is effective in removing carbamazepine from potable-water supplies (Ternes et al., 2002), whereas results of this study indicate that carbamazepine and other hydrophobic compounds such as AHTN and HHCB persist through DWT that includes filtration with GAC. Sorption efficiencies depend on competition with other organic 
compounds; therefore, the adsorption capacity for carbamazepine and other OWCs in a DWT facility that processes raw water that contains substantial amounts of many naturally occurring and anthropogenic organic compounds is expected to be smaller than that in laboratory and pilot-scale experiments in which fresh activated carbon and deionized water were used (Ternes et al., 2002). At the time of this study, the GAC filters in the DWT plant were approximately 3 years old and were used primarily to control odor- and tastecausing compounds in chlorinated water, with contact times ranging from approximately 1.5 to 3 min. Because previous investigators did not detect carbamazepine in potable-water supplies (Ternes et al., 2002), the presence of this compound in raw, filtered, settled, and finished water samples was verified using two additional mass spectrometric methods: high-performance liquid chromatography/tandem mass spectrometry with an ion-trap mass spectrometer (HPLC/IT-MS/MS; Bruker Daltonics Esquire) and high-performance liquid chromatography/time-of-flight mass spectrometry (HPLC/TOF-MS; Micromass LCT). In

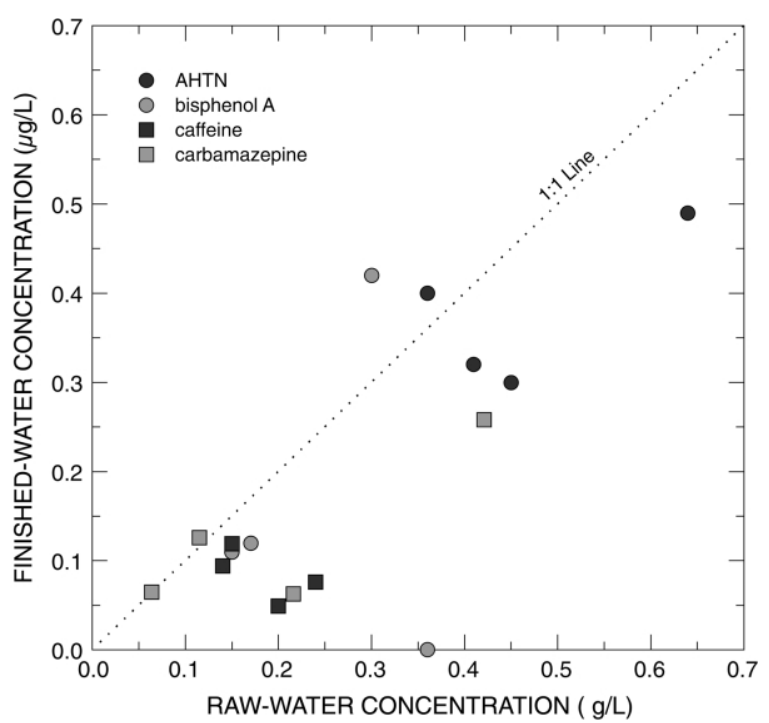

Fig. 4. Concentrations of selected compounds in samples of raw and finished water.

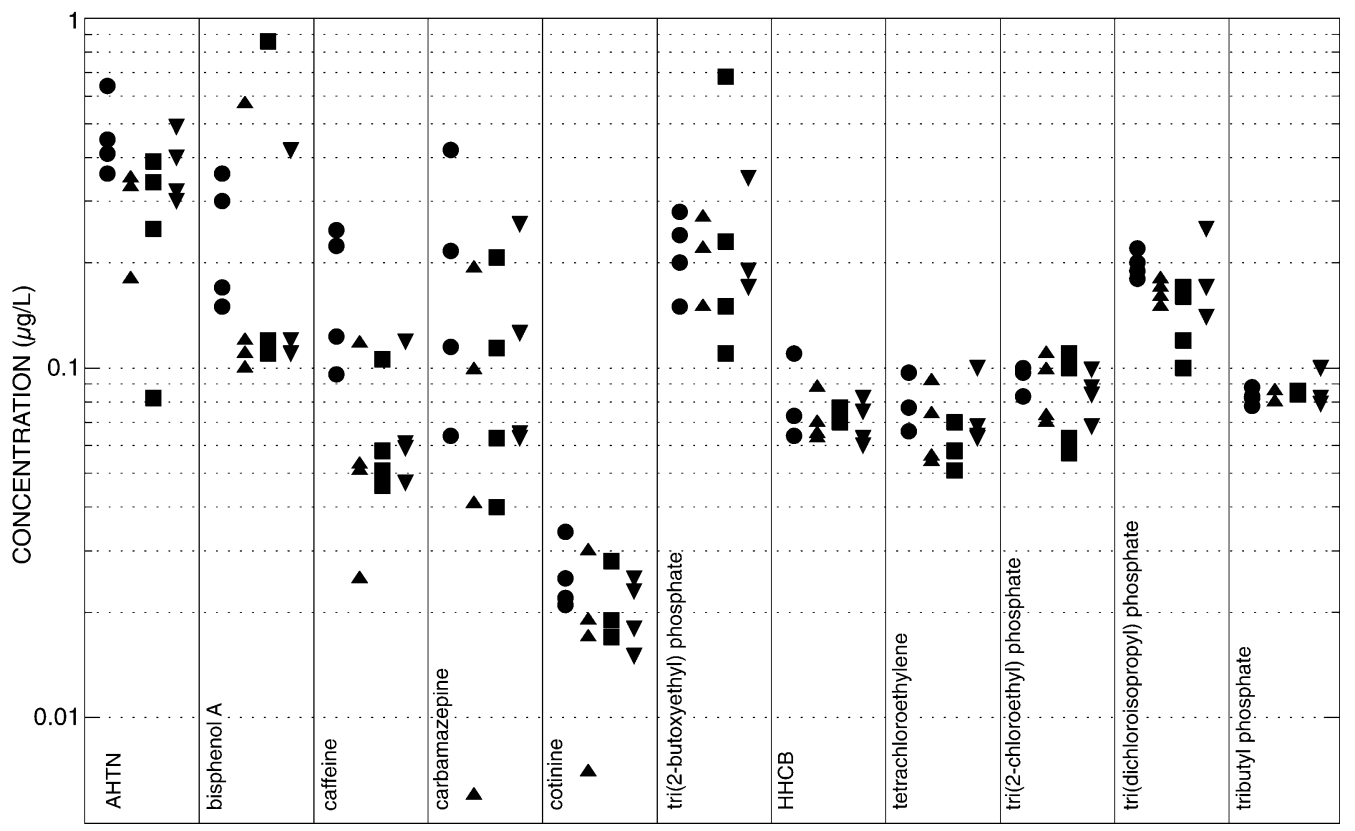

Fig. 3. Concentrations of selected compounds in samples of raw (circles), settled (triangles), filtered (squares), and finished (inverted triangles) water. 
all samples, HPLC/IT-MS/MS analysis of the putative carbamazepine peak in samples produced a protonated molecular ion at the retention of a carbamazepine standard. MS/MS analysis of this carbamazepine ion $(\mathrm{m} / \mathrm{z} 237)$ in all samples resulted in fragmentation yielding an ion of $m / z$ 194, reflecting a loss of $\mathrm{CONH}$, consistent with a loss and rearrangement of the carbamazepine protonated molecular ion. In all samples, the elemental composition of the suspected carbamazepine peak by HPLC/TOF-MS was consistent with the elemental composition of carbamazepine within 1.5 millidaltons. For the protonated molecular ion of carbamazepine, that converts to a mass accuracy error of no greater than 6 parts per million, well within the criteria commonly accepted for accurate mass determination. AHTN and HHCB detections in all samples were verified by full scan mass spectrometry analysis using careful comparison of each samples spectrum to a custom library spectrum made from authentic standards run on the same analytical instrument.

\section{Discussion}

Results of this study demonstrate that OWCs such as prescription and non-prescription drugs and their metabolites, fragrances, flame retardants, plasticizers, disinfectants, personal care products, detergent metabolites, products of oil use and combustion, and other extensively used chemicals are frequently detected in streams whose flow contains effluent from municipal STPs. These results corroborate those of Kolpin et al. (2002). Furthermore, this study demonstrates that some of these contaminants survive conventional DWT processes and occur in potable-water supplies, whereas others are reduced to non-detectable concentrations through conventional DWT processes. The occurrence of many of these contaminants in drinking water is unregulated and this study provides the first documentation of their occurrence in drinking-water supplies. This information can be used in setting research and regulatory priorities and in designing future monitoring programs.

Concentrations of the OWCs that were detected in finished water during this study generally were low $(93 \%<0.5 \mu \mathrm{g} / 1)$ (Fig. 2), and in the cases where standards have been established, did not exceed Federal drinking-water standards or lifetime health advisories. Most of these compounds, however, do not currently have established drinking-water standards or health advisories (Table 1); therefore, the potential health consequences associated with exposure through drinking water are not known. Concentrations in finished water of OWCs designed for human consumption, such as prescription and non-prescription drugs, were far below doses used in therapy. For example, the maximum possible intake of carbamazepine in finished water in a lifetime (assuming an intake of 21 per day for 70 years) was $13 \mathrm{mg}$, whereas a single therapeutic dose generally is $100 \mathrm{mg}$ or greater. Nevertheless, most studies on the therapeutic effects of drugs are based on the short-term ingestion of relatively high doses; little is known about potential health effects associated with longterm chronic ingestion of low concentrations through drinking water (Kümmerer, 2001). Moreover, drinking-water criteria currently are based on the toxicity of individual compounds and not combinations of compounds. The possibility that exposure to multiple organic compounds, even at low concentrations, may have a synergistic humanhealth consequence is an area of recent research (Birader and Rayburn, 1995; Marinovich et al., 1996), and the co-occurrence of organic compounds in drinking-water supplies has recently been documented (Stackelberg et al., 2001; Squillace et al., 2002). In this study, 11-17 different OWCs were detected in each of the four samples of finished water.

Whereas some frequently detected OWCs, such as pharmaceutical compounds, are designed to be ingested, others (e.g. flame retardants, solvents, and other personal care and industrial chemicals) are not designed for human consumption. The human-health consequence of chronic ingestion of these compounds is even less well understood than that of categories of OWCs such as pharmaceutical compounds. Some of these compounds (e.g. bisphenol A) are known or suspected endocrine disruptors and may be potent reproductive toxins even at low concentrations.

Although only a small number of degradate compounds were analyzed for in this study, several 
of the most frequently detected OWCs are degradates (e.g. cotinine, dehydronifedipine, erythromycin- $\mathrm{H}_{2} \mathrm{O}$, and 1,7-dimethylxanthine, degradates of nicotine, nifedipine, erythromycin, and caffeine, respectively); thus, the formation of degradates may represent a substantial component of the total transport of OWCs through DWT processes. This finding demonstrates the importance of analyzing for degradates in order to understand fully the fate of OWCs in a DWT facility as well as the potential human-health issues associated with chronic exposure to these compounds through drinking water. For other classes of organic compounds, such as pesticides, the importance of analyzing for degradates is well established (Thurman et al., 1994; Kolpin et al., 1997; Kalkhoff et al., 1998; Graham et al., 1999; Kolpin et al., 2001). The absence of many OWCs in finished-water supplies, therefore, does not necessarily imply their complete removal from finished water. Rather, the treatment process may transform parent OWCs to unknown and (or) unmeasured degradates. Additional methods development and sample analysis will be required to address these issues.

The limited number of samples $(n=4)$ collected at each site for this study and the fact that the collection of grab samples in the DWT plant did not adequately account for expected retention times throughout treatment preclude quantification of the effectiveness of each treatment process in reducing the concentrations of these contaminants. Additional sampling will provide information about (1) temporal variability of OWCs in source waters; (2) effectiveness of specific physical and chemical treatments in reducing the concentrations of the target OWCs; and (3) which primary physical processes and (or) chemical reactions reduce the concentrations of or eliminate OWCs. Only a small subset of the thousands of organic compounds that are currently in use and potentially could occur in domestic and industrial wastewaters were determined; however, this study indicates that at least some organic compounds can enter and persist in environmental waters and also may survive subsequent water-treatment processes. The technology to analyze for all known organic compounds is currently unavailable and, therefore, the complete extent of occurrence of OWCs in drink- ing-water supplies is unknown. The challenge for future studies is to develop the means to characterize the types and concentrations of these compounds that are likely to co-occur in drinking-water supplies and to assess their potential effects.

\section{Acknowledgments}

The authors thank the personnel of the watertreatment plant for permission to collect samples from their facility and for their cooperation with all aspects of this and related ongoing studies. This study was funded by the Centers for Disease Control and Prevention.

\section{References}

Barber LB, Brown GK, Zaugg SD. Potential endocrine disrupting organic chemicals in treated municipal wastewater and river water. In: Keith LH, Jones-Lepp TL, Needham LL, editors. Analysis of Endocrine Disruptors. ACS Symposium Series 747, Washington DC: American Chemical Society, 2000: pp. 97-123.

Birader DP, Rayburn AL. Chromosonal damage induced by herbicide contamination at concentrations observed in public water supplies. J Environ Qual 1995;24:1222.

Buser HR, Muller MD, Theobald N. Occurrence of the pharmaceutical drug clofibric acid and the herbicide mecoprop in various Swiss lakes and in the North Sea. Environ Sci Technol 1998a;32(1):188.

Buser HR, Poiger T, Muller MD. Occurrence and fate of the pharmaceutical drug diclofenac in surface waters: rapid photodegradation in a lake. Environ Sci Technol 1998b;32(22):3449.

Buser HR, Poiger T, Muller MD. Occurrence and environmental behavior of the chiral pharmaceutical drug ibuprofen in surface waters and in wastewater. Environ Sci Technol 1999;33(15):2529.

Cahill JD, Furlong ET, Burkhardt MR, Kolpin DW, Anderson LR. Determination of pharmaceutical compounds in surfaceand ground-water samples by solid-phase extraction and high-performance liquid chromatography/electrospray-ionization mass spectrometry. Accepted for publication, J Chromatogr A 2004.

Daughton CG. Pharmaceuticals and personal care products in the environment: overarching issues and overview. In: Daughton CG, Jones-Lepp TL, editors. Pharmaceuticals and personal care products in the environment: scientific and regulatory issues. ACS Symposium Series 791, Washington, DC: American Chemical Society, 2001.

Frick EA, Henderson AK, Moll DM, Furlong ET, Meyer MT. In: Hatcher KJ, editor. Proceedings of the 2001 Georgia Water Resources Conference, March 26-27, at The Univer- 
sity of Georgia, Athens, Georgia: Institute of Ecology, The University of Georgia, 2001.

Graham WH, Graham DW, Denoyelles F Jr, Smith VH, Larive CK, Thurman EM. Metolachlor and alachlor breakdown product formation patterns in aquatic field mesocosms. Environ Sci Technol 1999;33(24):4471.

Halling-Sorensen B, Nielsen SN, Lanzky PF, Ingerslev F, Lutzhoft $\mathrm{HCH}$, Jorgensen SE. Occurrence, fate and effects of pharmaceutical substances in the environment-a review. Chemosphere 1998;36(2):357.

Heberer T. Tracking persistent pharmaceutical residues from municipal sewage to drinking water. J Hydrol 2002;266:175.

Heberer TH, Stan HJ. Determination of clofibric acid and $\mathrm{N}$ (Phenylsulfonyl)-Sarcosine in sewage, river and drinking water. Int J Environ Anal Chem 1997;67:113.

Hignite C, Azarnoff DL. Drugs and drug metabolites as environmental contaminants: chlorophenoxyisobutyrate and salicyclic acid in sewage water effluent. Life Sci 1977;20(2):337.

Holm JV, Rugge K, Bjerg PL, Christensen TH. Occurrence and distribution of pharmaceutical organic compounds in the groundwater downgradient of a landfill, Grindsted, Denmark. Environ Sci Technol 1995;29(5):1415.

Kalkhoff SJ, Kolpin DW, Thurman EM, Ferrer I, Barcelo D. Degradation of chloroacetanilide herbicides: the prevalence of sulfonic and oxanilic acid metabolites in Iowa groundwaters and surface waters. Environ Sci Technol 1998;32(11):1738.

Kolpin DW, Kalkhoff SJ, Goolsby DA, Sneck-Fahrer DA, Thurman EM. Occurrence of selected herbicides and herbicide degradation products in Iowa's ground water, 1995. Ground Water 1997;35(4):679.

Kolpin DW, Thurman EM, Linhart SM. Occurrence of cyanazine compounds in groundwater: degradates more prevalent than the parent compound. Environ Sci Technol 2001;35(6):1217.

Kolpin DW, Furlong ET, Meyer MT, Thurman EM, Zaugg SD, Barber LB, Buxton HT. Pharmaceuticals, hormones, and other organic wastewater contaminants in US streams, 1999-2000: a national reconnaissance. Environ Sci Technol 2002;36(6): 1202 .

Kümmerer K. Introduction: pharmaceuticals in the environment. In: Kümmerer $\mathrm{K}$, editor. Pharmaceuticals in the environment: sources, fate, effects and risksSpringer, 2001. p. $1-8$.

Marinovich MR, Ghilard R, Gucci CL. Effect of pesticide mixtures on in-vitro nervous cells. Toxicology 1996;108:201.

National Research Council. Issues in potable reuse-the viability of augmenting drinking water supplies with reclaimed water. Washington, DC: National Academy Press, 1998. p. 263.

Reddersen K, Heberer T, Dunnbier U. Identification and significance of phenazone drugs and their metabolites in ground and drinking water. Chemosphere 2002;49:539.

Richardson ML, Bowron JM. Review-the fate of pharmaceutical chemicals in the aquatic environment. J Pharm Pharmacol 1985;37:1.

Shelton LR. Field guide for collecting and processing streamwater samples for the National Water-Quality Assessment Program. US Geological Survey Open-File Rep 94-455, 1994.

Squillace PJ, Scott JC, Moran MJ, Nolan BT, Kolpin DW. Volatile organic compounds, pesticides, nitrate, and their mixtures in groundwater used for drinking water in the United States. Environ Sci Technol 2002;36(9):1923.

Stackelberg PE, Kauffman LJ, Ayers MA, Baehr AL. Frequently co-occurring pesticides and volatile organic compounds in public supply and monitoring wells, Southern New Jersey, USA. Environ Toxicol Chem 2001;20(4):853.

Stumpf M, Ternes TA, Wilken RD, Rodrigues SV, Baumann W. Polar drugs in sewage and natural waters in the State of Rio de Janeiro, Brazil. Sci Total Environ 1999;225:135.

Ternes T. Pharmaceuticals and metabolites as contaminants of the aquatic environment. In: Daughton CG, Jones-Lepp TL, editors. Pharmaceuticals and personal care products in the environment: scientific and regulatory issues. ACS Symposium Series 791, Washington, DC: American Chemical Society, 2001.

Ternes TA, Meisenheimer M, McDowell D, Sacher F, Brauch H, Haist-Gulde B, Preuss G, Wilme U, Zulei-Seibert N. Removal of pharmaceuticals during drinking water treatment. Environ Sci Technol 2002;36(17):3855.

Thurman EM, Meyer MT, Mills MS, Zimmerman LR, Perry CA, Goolsby DA. Formation and transport of deethylatrazine and deisopropylatrazine in surface water. Environ Sci Technol 1994;28(13):2267.

Turner KS, Hardy MA, Tapper RJ. Water-quality reconnaissance of the perimeter of the Rolling Knoll landfill near Green Village, New Jersey, and electromagnetic survey of the parts of the landfill within the Great Swamp National Wildlife Refuge, 1989. US Geological Survey Open-File Rep 92-153, 1993.

US Geological Survey. National field manual for the collection of water-quality data. Techniques of Water-Resources Investigations, US Geological Survey Book 9, Handbooks for Water-Resources Investigations, 1998.

US Environmental Protection Agency. Drinking water standards and health advisories. US EPA No. 822-R-02-038; Washington, DC: US Government Printing Office, 2002. 